\title{
Conceituação da experiência de serviços por meio de uma revisão bibliográfica sistemática
}

\author{
Alexandre Meira de Vasconcelos \\ Programa de Pós-Graduação em Engenharia de Produção - UFSC \\ alexandre.meira@deps.ufsc.br \\ Rodrigo Barichello \\ Programa de Pós-Graduação em Engenharia de Produção - UFSC \\ rodrigobarichello@gmail.com \\ Álvaro Guillermo Rojas Lezana \\ Departamento de Engenharia de Produção e Sistemas - UFSC \\ lezana@deps.ufsc.br \\ Fernando Antônio Forcellini \\ Departamento de Engenharia de Produção e Sistemas - UFSC \\ forcellini@deps.ufsc.br \\ Marcelo Gitirana Gomes Ferreira \\ Departamento de Engenharia de Produção e Sistemas - UFSC \\ marcelo.gitirana@gmail.com \\ Paulo Augusto Cauchick Miguel \\ Departamento de Engenharia de Produção e Sistemas - UFSC \\ cauchick@deps.ufsc.br
}

\section{RESUMO}

As operações de serviços vêm assumindo elevada importância na atualidade com consequente aumento do interesse da academia pelo assunto. Nesse contexto, este artigo objetiva sistematizar os conceitos associados à experiência de serviços a partir de significados constatados na produção científica qualificada. Para tanto, inicialmente utilizou-se de uma busca sistemática na literatura para posterior análise bibliométrica visando identificar os principais artigos, periódicos, referências e palavras-chave. O estudo constata que a experiência de serviços e qualidade em serviços são conceitos distintos, devendo ser analisados separadamente. A construção do conceito proposto foi orientada pela análise de conteúdo dos artigos, na qual foram identificadas as dimensões e componentes que o categorizam de maneira singular. Enquadrou-se a experiência de serviços sob a metáfora de processos, com as dimensões de "predisposições", "interações" "e "reações", interdependentes, complementares, que condicionam a percepção da qualidade e a decisão de compra atual e futura. As experiências vividas pelos clientes necessitam uma análise destacada em face de sua importância estratégica para organizações prestadoras de serviço. Este trabalho ainda orienta para a realização de estudos empíricos para validar ou refutar o conceito proposto.

Palavras-chave: Experiência de serviços, Conceituação em serviços, Bibliometria.

\begin{abstract}
Service operations have assumed great importance in present with a consequent increase on the interest of academia in the subject. In this context, this article aims to systematize the concepts associated with the service experience from the meanings found at qualified scientific production. To do so, initially we used a systematic literature for further bibliometric analysis to identify the main articles, journals, references and keywords. The study found that the service experience and service quality are distinct concepts and should be analyzed separately. The construction of the proposed concept was driven by content analysis of articles, which identified the dimensions and components that categorize it in a singular way. Service experience was framed by the metaphor of processes, with the dimensions of "dispositions", "interactions" and "reactions", interdependent, complementary, which influence the quality perception and purchase
\end{abstract}


decision at present and future. The lived experiences by the clients need an highlighted analysis given its strategic importance to organizations that provide services. This study still enables to conduct empirical studies to validate or not the proposed concept.

Keywords: Service experience, Service conceptualization, Bibliometrics.

\section{Introdução}

As operações de serviços assumiram elevada importância no mundo contemporâneo com consequente aumento do interesse da academia pelo assunto (CHASE; APTE, 2007). Heineke e Davis (2007) destacam a importância do tema ao afirmarem a interdependência entre bens e serviços, ou seja, de que a produção e venda de produtos estão geralmente associadas a algum tipo de prestação de serviço (transporte, entrega, montagem, assistência, reposição, telemarketing, por exemplo). As observações de Bateson e Hoffman (2001) acrescentam que não há serviços ou produtos "puros" e que é possível evidenciar a coexistência de ambos nas relações de negócio em organizações industriais, comerciais ou de serviços.

A complexidade deste tipo de estudo reside nas características típicas dos serviços: intangibilidade, perecibilidade, heterogeneidade e simultaneidade, já estabelecidas na literatura (LOVELOCK; GUMMESSON, 2004; FITZSIMMONS; FITZSIMMONS, 2005; SLACK et al., 2006; CHASE; APTE, 2007; LADHARI, 2009; ZEHRER, 2009), todas com forte caráter subjetivo e de difícil mensuração. A simultaneidade, em particular, significa que a prestação do serviço e o consumo acontecem ao mesmo tempo o que a torna uma experiência única para cada cliente, bem como condiciona a percepção da qualidade e a decisão de compra atual e futura. Pine II e Gilmore (2002) apontam a experiência positiva dos clientes como um fator de diferenciação no posicionamento competitivo das organizações e, por este motivo, a experiência de serviços, seja virtual ou presencial, precisa ser estudada em maior profundidade com o intuito de entender sua natureza e para que se possam criar bases teóricas para instrumentos de medição e melhoria.

Nesse contexto, este trabalho tem como objetivo propor um conceito para a experiência de serviços por meio da consulta à produção científica qualificada. A presente proposta é de caráter teórico-conceitual, mas esperase que, futuramente, sirva como base para estudos empíricos, uma vez que não foram evidenciados na literatura consultada estudos deste tipo. Considera-se que a proposição de um conceito que traduza o significado amplo da experiência de serviços e que permita operacionalizá-lo em trabalhos futuros de natureza qualitativa ou quantitativa seja relevante para comprovar ou refutar as abordagens identificadas.
Constatam-se na literatura (BARON; HARRIS, 2010; JOHNSON et al., 2009; JOHNSTON; KONG, 2011; KIM, S. et al., 2011; TENG, 2011; YI; GONG, 2009) algumas propostas de avaliação da experiência de serviços que a colocam como uma extensão da satisfação dos clientes ou da qualidade em serviços. No entanto, não são identificadas propostas que tratam a experiência de serviços como objeto central de análise.

Desse modo, surge então uma questão central de pesquisa: “o que é a experiência de serviços?". Esta questão remete à proposição central de que a experiência de serviços não se traduz somente como consequência da prestação do serviço, mas depende de outros fatores tais como: os atributos dos atores envolvidos, o contexto social, cultural e ambiental, da forma como o serviço é prestado e da natureza deste serviço. Helkkula (2011) conclui que, em estudos empíricos, há três caracterizações típicas, sob as quais a produção científica aborda a experiência de serviços: nas situações ou fenômenos vividos; nos processos operacionais; e nos resultados ou efeitos da prestação; e ainda sugere a possibilidade de complementaridade entre elas.

A fim de atingir o objetivo estabelecido, o presente trabalho é dividido em quatro seções, além desta introdução. A próxima seção descreve as técnicas de pesquisa e métodos adotados, enquanto que a seção seguinte apresenta os resultados do estudo bibliométrico do portfólio de artigos selecionados. A seção subsequente apresenta o conceito e a explanação dos termos que compõem a experiência de serviços, fundamentando-os com base na análise de conteúdo por meio da leitura completa dos artigos. A última seção traz as conclusões e recomendações para trabalhos futuros.

\section{Métodos e Técnicas de Pesquisa}

A base de investigação e da análise é a literatura qualificada obtida por uma revisão bibliográfica sistemática, conduzida em três etapas (WALSHAM, 2006; LEVY; ELLIS, 2006): i) procedimento de busca e seleção de artigos; ii) análise de resultados (bibliometria, leitura e análise de conteúdo); iii) documentação (definição do conceito de experiência de serviços).

Uma premissa para este trabalho é que o conceito de experiência de serviços está ancorado em uma visão sistêmica de uma organização sob a metáfora de organismo que interage com o meio e dele sofre pressões e 
sobre ele exerce influência em um processo dinâmico e plural (CALDAS, 2005; MORGAN et al., 1996). A metáfora da experiência de serviços como um processo estimula naturalmente a decomposição em três dimensões de análise: requisitos de entrada (inputs), os subprocessos de transformação e os resultados (outputs) (GONÇALVES, 2000; SLACK et al., 2006; ANDERSEN, 2007). A abordagem por processos também proporciona uma estrutura que facilita a análise, controle, gestão e melhoria das atividades e possibilita alcançar, manter ou aperfeiçoar o desempenho dos negócios (KUMAR et al., 2008; SMART et al., 2009; BEIMBORN; JOACHIM, 2011; BITITCI et al., 2011).

\subsection{Busca, Recuperação e Seleção de Artigos}

A primeira ação corresponde à seleção das bases de dados a serem consultadas, as palavras-chave para realização da busca e a definição do recorte temporal. Optou-se por selecionar artigos científicos coletados nas seguintes bases de dados: Scopus e Web of Science, com a palavra-chave "service experience", sem delimitações ao ano de publicação. A escolha dessas bases é devida a sua importância e por proporcionar uma ampla taxa de cobertura de artigos nas áreas de engenharia de produção e administração, resultando em uma quantidade significativa de artigos para análise posterior. Além disso, essas duas bases permitem maior facilidade de acesso e exportação das informações dos artigos para posterior análise por software especializados no tratamento de metadados. Para gerenciamento dos metadados foi adotado o EndNote ${ }^{\circledR}$ e para a geração de mapas de citação e co-citação o Sci2Tool ${ }^{\circledR}$ e o Guess ${ }^{\circledR}$. Estes últimos programas oferecem facilidade na manipulação das informações e uma interface gráfica clara e objetiva. Os metadados, transferidos para o EndNote ${ }^{\circledR}$, são eliminados e/ou tratados, bem como os artigos em duplicidade e aqueles cujo título e resumo mostrem uma falta de aderência ao escopo do presente trabalho, isto é, não oferecem contribuição no tema investigado, identificada por meio da leitura do artigo. Os metadados incompletos ou que apresentam erros são corrigidos e, posteriormente é realizado um download dos artigos/ arquivos completos, geralmente disponíveis em formato pdf, cujo acesso seja livre e gratuito. Para completar o registro dos dados, anexa-se estes arquivos aos respectivos metadados no software EndNote ${ }^{\circledR}$.

\subsection{Análise dos Resultados}

A análise da literatura é dividida em duas partes: análise bibliométria e análise de conteúdo. A primeira parte é uma avaliação das publicações, de cunho mais quantitativo no sentido de apontar alguns indicadores, tais como os artigos mais representativos, os periódicos em que são publicados e palavras-chave para situar o termo na produção científica qualificada, apresentandoos em tabelas e mapas, quando apropriado. A segunda parte é de natureza mais qualitativa consistindo na investigação sobre os conteúdos literalmente expressos e aqueles latentes ou subentendidos nos escritos de outros pesquisadores (FRANCO, 2007). Realiza-se, portanto, uma leitura, análise e interpretação dos textos para identificar elementos que auxiliem na construção do conceito proposto. Os resultados desta análise são resumos e resenhas sobre os textos estudados. Esta parte também inclui a organização dos textos nas dimensões de entradas, processos de transformação e saídas, conforme a metáfora estabelecida.

\subsection{Determinação do Conceito Proposto sobre Experiência de Serviços}

Nesta etapa busca-se nos textos como os autores definiram/conceituaram experiência de serviços independentemente de terem recorrido a outras fontes. Visando discutir estes resultados, outras fontes adicionais poderão ser usadas para explicitar o conceito, quando apropriado. Algumas dimensões são também identificadas. Estas derivam do conceito mais amplo, categorizamno em critérios mais específicos de análise e podem ser decompostas em componentes, caso seu nível de complexidade seja elevado. No processo de seleção, análise e construção do conhecimento, buscar-se então definir um conceito sistêmico (proposto), elaborado e situado nos conceitos e paradigmas respaldados na literatura qualificada que permita ser testado empiricamente, conforme estabelecido por Campenhoudt e Quivy (2003). O conceito é estabelecido por análise do conteúdo das dimensões, embasados na literatura, no intuito de incorporar seus aspectos essenciais das publicações.

\section{Análise Bibliométrica}

Esta seção apresenta os resultados mais relevantes do estudo bibliométrico para situar a pesquisa do ponto de vista da evolução da produção qualificada sobre a experiência de serviços, bem como identificar os artigos, periódicos e palavras-chave mais relevantes. Como mostra o Gráfico 1, as publicações iniciaram em 1991 e vêm crescendo desde então, particularmente nos últimos anos. 


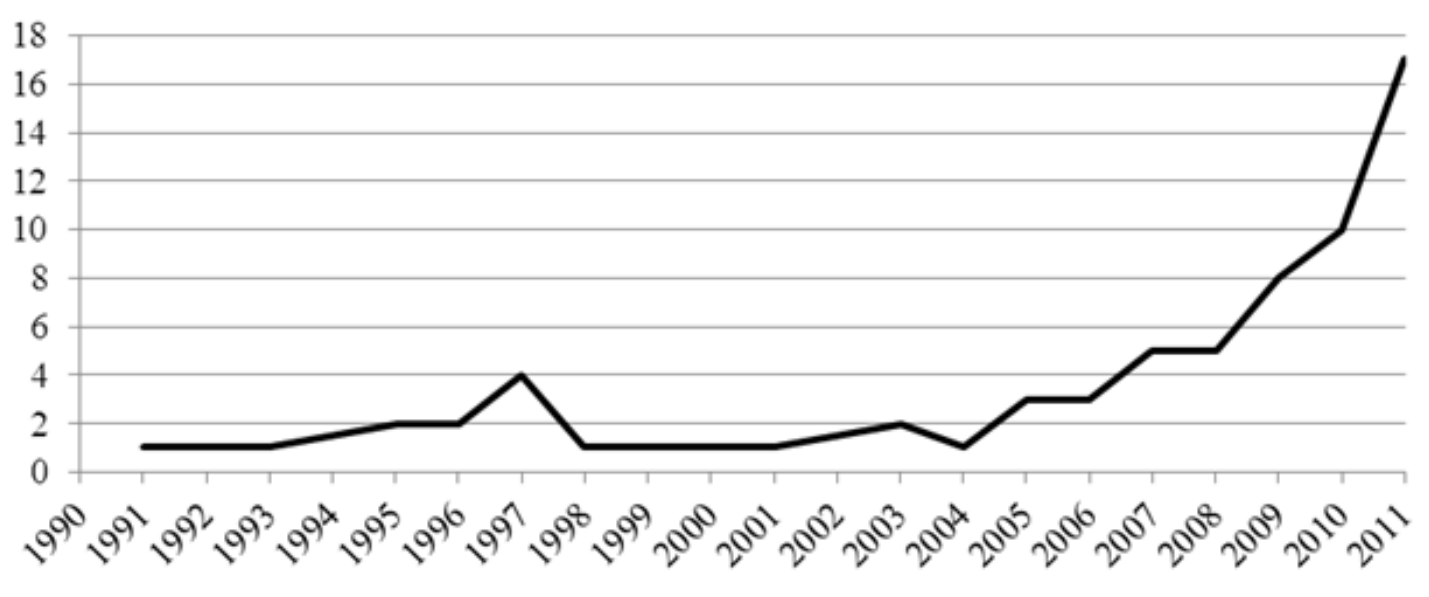

Gráfico 1 - Evolução da produção científica sobre experiência de serviços

Um incremento nas publicações ocorre a partir de 2003 e tem aumentado desde então, o que sugere aumento de interesse sobre este tema. Anteriormente, houve um pico de produção em 1997, mas isso pode ser explicado pelo fato da publicação de trabalhos (GROVE; FISK, 1997; WINSTED, 1997) que compuseram uma edição especial do periódico Journal of Retailing sobre perspectivas de excelência em serviços.

Quanto às palavras-chave, observou-se uma forte relação de proximidade entre estudos sobre experiência de serviços relacionados à qualidade em serviços (ver Figura 3). As duas palavras-chave mais presentes nos artigos são "quality" e "satisfaction" que também aparecem, com o mesmo sentido, em outras palavraschave compostas (customer satisfaction; service quality, consumer satisfaction; customer service quality). As palavras-chave "experience" e "service experience" aparecem somente em artigos dos últimos três anos (2009-2011) o que sugere ser recente o tratamento da experiência de serviços com maior especificidade.

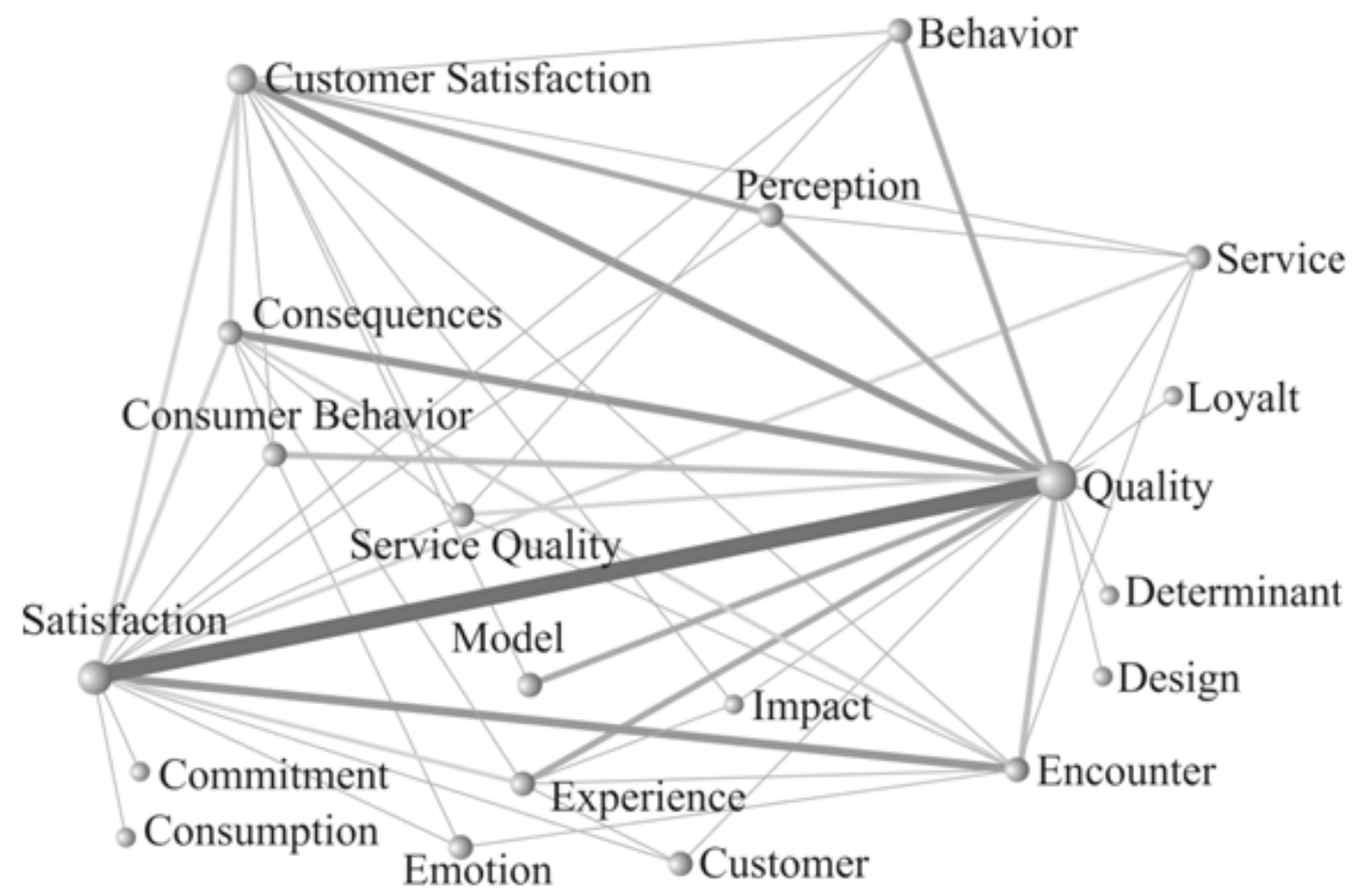

Figura 2 - Mapa de cocitações das palavras-chave. 
Entre os periódicos de destaque no portfólio do total de artigos, destacam-se os que têm fator de impacto maior que 1,0. Em ordem decrescente de fator de impacto, os periódicos mais relevantes são: Journal of Operations Management (5,093); Journal of Consumer Research (2,590); Journal of Retailing (2,257); International Journal of Service Industry Management $(1,800)$; Journal of Business Research (1,773); Journal of Service Research (1,615); Service Industries Journal (1,071). Cabe destacar que os artigos mais citados do Quadro 1 foram publicados nestes periódicos de destaque.

Do portfólio de 70 publicações identificadas nos últimos vinte anos (1991 a 2012), analisaram-se inicialmente os artigos mais citados (Tabela 1), com um número mínimo de 24 citações e um máximo de 169, com média de mais de cinco citações por ano.

Tabela 1 - Artigos do portfólio com maior número de citações na Web of Scien

\begin{tabular}{l|c}
\hline \multicolumn{1}{c|}{ Artigo } & $\begin{array}{c}\text { No citaç̃̃es } \\
\text { Web of Science }\end{array}$ \\
\hline Edvardsson; Enquist; Johnston (2005) & 24 \\
\hline Froehle; Roth (2004) & 50 \\
\hline Boshoff; Leong (1998) & 30 \\
\hline Bitner; Booms; Tetreault (1997) & 73 \\
\hline Grove; Fisk (1997) & 71 \\
\hline Winsted (1997) & 66 \\
\hline Bettencourt; Gwinner (1996) & 32 \\
\hline Johnston (1995) & 35 \\
\hline Kingman-Brundage; George; Bowen (1995) & 24 \\
\hline Hui; Bateson (1991) & 169 \\
\hline
\end{tabular}

Os artigos mais referenciados nos artigos do portfólio são apresentados na Tabela 2. Nestes artigos há um reforço da ideia de que a experiência de serviços é um extrato da qualidade dos serviços, mas será visto mais adiante que se trata de uma visão equivocada.

Tabela 2 - Artigos mais utilizados como referências nos artigos do portfólio

\begin{tabular}{l|c|c}
\hline \multicolumn{1}{c|}{ Artigo } & $\begin{array}{c}\mathbf{N}^{\mathbf{0}} \text { citações } \\
\text { Web of Science }\end{array}$ & $\begin{array}{c}\mathbf{N}^{\mathbf{0}} \text { citações } \\
\text { no Portfólio }\end{array}$ \\
\hline Vargo; Lusch (2004) & 790 & 14 \\
\hline Zeithaml; Berry; Parasuraman (1996) & 987 & 13 \\
\hline Bitner (1992) & 555 & 15 \\
\hline Cronin Jr; Taylor (1992) & 1.018 & 17 \\
\hline Bitner; Booms; Tetreault (1990) & 712 & 31 \\
\hline Parasuraman; Zeithaml; Berry (1988) & 2.071 & 14 \\
\hline Parasuraman; Zeithaml; Berry (1985) & 1.954 & 18 \\
\hline
\end{tabular}

Os artigos mais recentes, de 2009 a 2012 também foram estudados por trazerem o que há de mais novo sobre o assunto (Tabela 3), mesmo não tendo ainda como avaliar o número de citações, uma vez que não houve tempo hábil para que fossem citados em outros artigos.

Tabela 3 - Artigos mais recentes sobre experiência de serviços

\begin{tabular}{l|c}
\multicolumn{1}{c|}{ Artigo } & $\begin{array}{c}\mathbf{N}^{\mathbf{0}} \text { citações } \\
\text { Web of } \text { Science }\end{array}$ \\
\hline He; Chen; Alden (2012) & 00 \\
\hline Gross; Pullman (2012) & 00 \\
\hline Helkkula (2011) & 00 \\
\hline Kim, J. et al. (2011) & 00 \\
\hline \multicolumn{2}{c}{ (continua...) }
\end{tabular}

(... continuação)

\begin{tabular}{l|l}
\hline Svari et al. (2011) & 00 \\
\hline Allred; Money (2010) & 00 \\
\hline Bryson; Taylor (2010) & 04 \\
\hline Chang; Horng (2010) & 00 \\
\hline Finsterwalder; Tuzovic (2010) & 02 \\
\hline Kwortnik; Thompson (2009) & 02 \\
\hline Ladhari (2009) & 02 \\
\hline Voorhees et al. (2009) & 01 \\
\hline Zehrer (2009) & 00 \\
\hline
\end{tabular}

\section{Conceituação da Experiência de Serviços}

Após a análise bibliométrica anteriormente apresentada, realizou-se uma análise mais aprofundada do conteúdo dos artigos em busca de conceitos, definições ou abordagens adotados pelos autores em relação à experiência de serviços. O propósito principal foi a construção de um conceito ampliado do termo. A análise do conteúdo se restringiu a três conjuntos de artigos: os artigos do portfólio com mais citações na Web of Science; os mais recentes; e os mais utilizados como referências nos artigos do portfólio.

Uma das constatações identificadas na literatura é a de que a experiência de serviços e a prestação de serviços são conceitos distintos (BRYSON; TAYLOR, 2010), ou seja, fornecer o serviço é a ação da organização para os clientes em uma complexa interligação de pessoas e infraestrutura por meio de processos estabelecidos (KWORTNIK; THOMPSON, 2009), e a experiência é singular a cada cliente o que se traduz em algo único e não generalizável (GONZÁLEZ; COMESAÑA; BREA, 2007).

As necessidades e valores do cliente são inputs da experiência de serviços, visto que é um fenômeno que se opera internamente (CHANG; HORNG, 2010), assim como o contexto social, cultural e ambiental em que a situação da prestação de serviços está inserida (GROSS; PULLMAN, 2012; ALLRED; MONEY, 2010). Sob esta ótica, o cliente é o locus onde a experiência de serviços se processa e não somente um recurso a transformar. No exemplo de um indivíduo que vai ao dentista para um tratamento, a dor que sente e/ou o medo que tem de anestesia são predisposições que podem tornar a experiência mais ou menos satisfatória e independem da qualidade profissional do dentista, do estado odontológico do paciente ou do ambiente em que este serviço é prestado.

Alguns autores afirmam que a experiência de serviços ocorre na situação vivida (ALLRED; MONEY, 2010; HELKKULA, 2011; VOORHEES et al., 2009) e, embora pareça trivial apontar o caráter vivencial da experiência de serviços, afirma-se que ela é vivida e construída antes, durante e após a prestação de serviço. Por isso, compreender os mecanismos deste episódio e criar meios para medi-lo pode se converter em vantagem competitiva para a organização (CHEN; CHEN, 2010). 
Com base na literatura, elaborou-se o conceito de experiência de serviços incluindo a noção de processo, e suas dimensões e componentes.

O conceito foi elaborado com três dimensões chamadas de "predisposições" (entradas), "interações" (transformações) e "reações" (saídas), respeitando a metáfora da experiência de serviços como um processo (Quadro 1). As organizações de serviços não têm controle sobre primeira e a última dimensão, pois estas são inerentes aos clientes e podem mudar conforme o momento ou a situação vivida. Há, no entanto, a possibilidade de a empresa intervir nas predisposições e reações usando-se do marketing ou de técnicas de decoração de ambientes (em lojas, por exemplo) para induzir os clientes a comportamentos positivos (TOSUN, 2007). A experiência de serviços é consequência das operações, das características organizacionais e pessoais (BRUNNER-SPERDIN; PETERS, 2009). Neste sentido, a experiência é um fenômeno pessoal e integral (NEAL; GURSOY, 2008) que incorpora as percepções e o nível de satisfação com cada aspecto do serviço, sejam internos ou externos à organização ou aos indivíduos.

\begin{tabular}{|c|c|c|c|c|c|}
\hline CONCEITO & \multicolumn{5}{|c|}{$\begin{array}{l}\text { É um processo cognitivo, afetivo e comportamental, vivido na prestação de serviços, que constrói, } \\
\text { solidifica ou modifica a imagem da organização e subsidia a decisão de compra atual ou futura dos } \\
\text { clientes; mediado por pessoas, infraestrutura e tecnologias; influenciado por valores e necessidades } \\
\text { dos clientes, pelo contexto sociocultural e ambiental, por experiências anteriores e pela natureza e } \\
\text { duração das operações de serviço. }\end{array}$} \\
\hline DIMENSÕES & PREDISPOSIÇÕES & & INTERAÇÕES & & REAÇÕES \\
\hline COMPONENTES & $\begin{array}{l}\text { Contexto sociocultural } \\
\text { Contexto ambiental } \\
\text { Imagens preconcebidas } \\
\text { Experiências anteriores } \\
\text { Traços de personalidade } \\
\text { Necessidades } \\
\text { Valores } \\
\text { Crenças } \\
\text { Habilidades }\end{array}$ & $\Rightarrow$ & $\begin{array}{l}\text { Staff da empresa } \\
\text { Infraestrutura } \\
\text { Tecnologias } \\
\text { Duração do evento } \\
\text { Natureza das operações }\end{array}$ & $\Rightarrow$ & $\begin{array}{l}\text { Comportamentos } \\
\text { Sentimentos } \\
\text { Aprendizado } \\
\text { Percepção de valor } \\
\text { (re)construção da imagem } \\
\text { Intenções } \\
\text { Desejos }\end{array}$ \\
\hline
\end{tabular}

Quadro 1 - Conceito de experiência de serviços, suas dimensões e componentes

A primeira dimensão de análise foi nomeada de "predisposições" por incorporar as disposições antecipadas ou tendências do cliente estar apto, desejoso, com imagens pré-concebidas, e levar em conta suas experiências anteriores nesse ou em outros negócios, além de suas necessidades e valores.

"Interações" foi o nome atribuído a segunda dimensão para distinguir claramente o instante da prestação do serviço, ou seja, quando o cliente faz um contato físico ou virtual com a organização e quando ocorrem as interações do cliente com o ambiente físico, tecnológico e com os recursos humanos da empresa.

A última dimensão, "reações", foi assim chamada fazendo uma analogia com uma reação química na produção de novos componentes, do que na reação física de oposição de dois corpos. As experiências modificam o cliente, provocam emoções, decisões, desejos, ações, aprendizado, e percepções que solidificam ou desconstroem a imagem da organização.

As reações, em um processo cíclico, transformam-se em predisposições para relacionamentos futuros com a organização. Assim sendo, manejar e gerenciar os espaços experienciais antes, durante e após a prestação do serviço torna-se um desafio para os gestores de serviços que desejem melhorar seu posicionamento competitivo.
A dimensão em que a gerência tem mais ação em termos de gestão e influência é a de "interações", na situação vivida no dia-a-dia nas quais há um maior contato do cliente com a organização. Entretanto, esforços de marketing são empreendidos para gerar necessidades e modificar valores dos clientes ou para minimizar históricos de prestação de serviços de baixa qualidade ou desconstruir imagens negativas, ambos a um custo normalmente elevado. Estas dimensões são discutidas em mais detalhes na sequência.

\subsection{Predisposições}

A experiência de serviços é o resultado de imagens préconcebidas ou construídas em experiências anteriores (ALLRED; MONEY, 2010; LADHARI, 2009), mas igualmente constrói, consolida ou modifica tais imagens (FINSTERWALDER; TUZOVIC, 2010; SVARI et al., 2011; TORRES JÚNIOR; MIYAKE, 2011). A imagem é, ao mesmo tempo, insumo e resultado da experiência (LADHARI, 2009; BOSHOFF; LEONG, 2008) e mesmo que alguém nunca tenha ouvido falar na empresa ou nunca tenha nela entrado, a disposição em contatá-la para uma possível prestação de serviço significa que já construiu uma imagem que o predispõe a passar pela experiência. A motivação pode ter originado na imagem construída por um marketing organizacional adequado, pela necessidade e valores do cliente, pela 
localização do negócio, pela falta de opções ou por uma série de outros fatores isolados ou combinados (RYU; HAN; KIM, 2008). No caso de hotéis, por exemplo, as informações imprecisas e inconsistentes no momento da reserva explicam a diferença entre as expectativas ou imagem pré-concebida e a experiência vivida, ou imagem construída, com severas consequências para o negócio (SUTHERLAND; DRUMMOND, 2007; ALVAREZ, et al.; 2008).

As entradas ou inputs da experiência de serviços correspondem a um conjunto de predisposições do cliente, simbolizadas por vários componentes. Não são estanques e imutáveis, estão fortemente ligadas à subjetividade do cliente e, portanto, são suscetíveis a influências do estado de humor, clima da região, relações do indivíduo com o consumo, pré-julgamentos, estado emocional, estado de saúde, suas necessidades, valores, religiosidade, entre outros (CHANG; HORNG, 2010; FINSTERWALDER; TUZOVIC, 2010).

As necessidades e valores dos atores envolvidos, sejam clientes ou colaboradores da empresa, constroem parte da experiência (CHANG; HORNG, 2010), pois juntos são os elementos que potencializam a motivação ou desmotivação para viver a experiência de forma mais ou menos intensa. (ALLRED; MONEY, 2010). O contexto sócio-cultural e ambiental é de profunda relevância para a experiência de serviços, uma vez que afetam as respostas dos clientes, assim como o tempo de espera real ou percebido na prestação do serviço (ALLRED; MONEY, 2010; LADHARI, 2009; PATRICIO; FISK; CUNHA, 2008). Geralmente, não pode ser modificado pela organização, mas o alcance de sua influência deve ser compreendido e devem ser tomadas ações para eliminar ou minimizar seus efeitos. Na noção de contexto está compreendida a singularidade da organização avaliada, o momento histórico, o reconhecimento da marca e aspectos regionais.

Frente à pluralidade de representações e significados que um indivíduo dá às situações vividas, a experiência de serviços pode ser extremamente satisfatória em determinadas circunstâncias e em outras não (SVARI et al., 2011; LADHARI, 2009). Porém, os clientes vêm com predisposições que impactam na experiência de serviços (DE ROJAS; CAMARERO, 2008; YEUNG; LEUNG, 2007). Indivíduos arrojados, por exemplo, terão maior predisposição a experimentar serviços com elevado nível de exposição pessoal e, este traço de sua personalidade não muda com facilidade.

\subsection{Interações}

As interações são as atividades e recursos mobilizados pela organização na prestação dos serviços que transformam predisposições em reações. Encontramse referências a estes espaços e instantes de interação como encontros de serviços (ALLRED; MONEY, 2010; FINSTERWALDER; TUZOVIC, 2010; CRONIN JR; TAYLOR, 1992), momentos da verdade (SVARI et al., 2011; ZEHRER, 2009), ou incidentes críticos (CHANG; HORNG, 2010; VOORHEES et al., 2009; BITNER; BOOMS; TETREAULT, 1990). Estas interações são afetadas pela complexidade das operações de serviço, o tempo de duração real e percebido do serviço, o nível e qualidade dos contatos entre clientes e destes com o staff organizacional, com as tecnologias, com a infraestrutura e com o sistema de informações (BASSANI, 2008). Gopalan e Narayan (2010) classificaram os componentes mediadores e influenciadores da experiência de serviços em: extrínsecos (incluindo as políticas de governo e infraestrutura de cidades) e intrínsecos, sob o domínio dos gestores das organizações prestadoras de serviços.

A experiência de serviços é mediada ou moderada por pessoas, tecnologias e infraestrutura (FROEHLE, ROTH, 2004; LADHARI, 2009; PATRICIO; FISK; CUNHA, 2008) que, neste caso, são o próprio cliente, o staff da empresa (BETTENCOURT, 1996) ou os demais clientes principalmente quando compartilham da prestação de serviço ativa ou passivamente, exemplificados em passeios turísticos, filas de banco, hospitais, para citar algumas situações (GROVE, FISK, 1996; FINSTERWALDER, TUZOVIC, 2010).

Há empresas em que é imperativo o cliente entrar no ambiente de serviço para tomar a decisão de compra. O contato com os elementos tangíveis do ambiente constroem a percepção da imagem corporativa que contaminarão positiva ou negativamente a experiência vivida (BRUNNER-SPERDIN; PETERS; STROBL, 2012). Quanto à interação cliente-colaborador, Boshoff e Leong (1998) apontam que pode ser aprimorada se os colaboradores tomarem para si os problemas dos clientes, os solucionarem e se desculparem pelas possíveis inconveniências ocorridas. Um fator de mediação da experiência de serviços é o grau de envolvimento, conhecimento e habilidade dos clientes com as tecnologias (PATRICIO; FISK; CUNHA, 2008) e estas, em alguns tipos de serviço, interferem fortemente na experiência tais como em sistemas de autoatendimento de bancos, compras pela internet, lan houses, entre outros. Dependendo do nível cultural, faixa etária e dos níveis de analfabetismo funcional, a prestação do serviço somente se torna viável com a interferência direta do pessoal da empresa, de forma a tornar sua experiência menos traumática.

\subsection{Reações}

Chen e Chen (2010) definem a experiência de serviços como resultado do consumo ou uso de um serviço manifestado por sensações e reações subjetivas. A experiência predispõe o cliente a reações (outputs) 
mais complexas que a mera constatação da qualidade do serviço ou percepção de valor. A experiência de serviços é um processo ou uma cadeia sequencial lógica que converte recursos em um resultado em forma de sentimentos, emoções, aprendizados e intenções. As emoções e aprendizados durante o consumo podem ser explícitas ou silenciosas, conscientes ou inconscientes, focadas em uma determinada situação (medo, raiva, alegria) ou gerais (positivas, neutras ou negativas) e marcam, mediam e moderam as decisões do cliente em consumir e norteiam o consumo atual e futuro (SVARI et al., 2011; LADHARI, 2009; HUTCHINSON; LAI; WANG, 2009).

Uma reação, no sentido aqui exposto, não significa necessariamente que há uma ação do sujeito, mas uma (re)significação do conjunto de predisposições de entrada, atribuindo-lhes uma estatura maior, menor ou igual ao que era antes da experiência e se tornam as predisposições para contatos futuros com a organização.

A experiência de serviços também é vista como um momento de aprendizado para o cliente e para a organização, pois, neste instante, formam-se juízos a respeito da organização, da qualidade da prestação do serviço, da percepção de valor (CHANG; HORNG, 2010; LADHARI, 2009; PATRICIO; FISK; CUNHA, 2008; VOORHEES et al., 2009). Os comportamentos e emoções advindos da experiência de serviços resultam dos desejos e expectativas do cliente ou induzidos pelo seu grupo social ou aplicados do exterior por algum outro agente, fatores subjetivos amplamente observados na literatura (CHANG; HORNG, 2010; LADHARI, 2009; SVARI et al., 2011; VOORHEES et al., 2009).

As reações mais polarizadas do cliente são o encantamento, que é importante para o negócio, ou a frustração, que é exatamente o oposto, mas outras de maior ou menos magnitude também são observadas. Contudo, não seria adequado afirmar que toda experiência ou é encantadora ou frustrante, pois tal maniqueísmo não foi observado na literatura de referência, em que é comum o uso de escalas graduais para medir a experiência de serviços (MIKULIĆ; PREBEŽAC, 2011). Obviamente que pode haver reações explícitas em forma de reforço à experiência satisfatória ou o repúdio à insatisfatória, mas o que se considera como relevante são os aspectos subjetivos e intersubjetivos das reações que, em alguns casos, predispõem o cliente à ação. Incluem-se neste escopo os fatores cognitivos, afetivos, comportamentais, simbólicos e aqueles que comporão o conjunto de intenções do cliente após viver a experiência (GROSS; PULLMAN, 2012; HÖGSTRÖM; ROSNER; GUSTAFSSON, 2010; MARTIN-RUIZ; CASTELLANOSVERDUGO; OVIEDO-GARCIA, 2010).

Uma das necessidades para as prestadoras de serviço é tornar a experiência de serviços diferenciada e adaptada às individualidades dos clientes (HÖGSTRÖM; ROSNER; GUSTAFSSON, 2010) e isso implica em uma leitura diferenciada sobre os negócios e em conhecer, medir e compreender a experiência vivida. A literatura ainda coloca que devem-se integrar apreciações cognitivas e emocionais para obter mais informação sobre a satisfação e comportamento do consumidor (MARTIN-RUIZ; CASTELLANOS-VERDUGO; OVIEDO-GARCIA, 2010). O modelo SERVQUAL (PARASURAMAN; ZEITHAML; BERRY, 1988) é um instrumento para mensuração da qualidade em serviços genérico amplamente empregado, mas é questionado por Huang et al. (2012) o fato do instrumento desconsiderar os fatores afetivos e o ambiente endógeno e exógeno que contribuem para compreender o fenômeno experiencial. Frente a isso, instrumentos para medir a experiência de serviços necessitam considerar não somente as expectativas e o desempenho, mas as predisposições - que são mais que expectativas - e também as interações e as reações - que são mais que a percepção do desempenho.

\section{Conclusões}

A experiência de serviços começou a ser tratada como um conceito distinto da qualidade da prestação de serviços nos últimos três anos, ainda que existam trabalhos que exploram as interfaces, com enfoque mais específico. Mesmo com o maior interesse no tema, identificado pelas publicações, não foi evidenciado um conceito explicíto na produção qualificada que abrangesse a amplitude e complexidade do tema.

No estudo bibliométrico, foi identificado um portfólio de 55 artigos nas bases de dados, onde foram discriminados os artigos, suas referências, periódicos e palavras-chave mais representativos. A análise do conteúdo desses artigos orientou a construção do conceito de experiência de serviços ancorado e explicitado em suas dimensões e componentes. Constata-se que que a experiência de serviços é um fenômeno de origem sistêmica que se processa individualmente e gera aprendizados, emoções e significados que mediam a relação do cliente com a organização.

A partir disso, o conceito de experiência de serviços assumiu a metáfora de um processo dividido nas dimensões de "predisposições", "interações" e "reações", interdependentes e complementares, que condicionam a percepção da qualidade da prestação do serviço e as relações atuais futuras com a organização. Tais dimensões suportam componentes objetivas, como o tempo de duração do serviço; subjetivas tais como sentimentos e emoções do cliente; e intersubjetivas, resultantes do relacionamento entre as pessoas que participam da prestação de serviços. A metáfora como recurso simbólico para comparar a experiência de serviços a um 
processo é adequada porque o conceito aqui tratado é teórico, bastante subjetivo, e que precisa ser explicitado, pois se pretende que seja compreendido, comunicado e disseminado. Conforme Bowdle e Gentner (2005), indivíduos submetidos a metáforas convencionais (de processo, por exemplo) compreendem mais facilmente o conteúdo associado.

O presente estudo defende que a experiência de serviços não é condicionada somente pela prestação do serviço, mas por um conjunto de fatores controláveis e outros fora do domínio da organização, porém passíveis de influência em alguns casos. O estudo ainda constata a importância de dissociar as pesquisas sobre experiência de serviços daquelas sobre qualidade de serviços. Os modelos usuais de avaliação da qualidade desconsideram aspectos afetivos, cognitivos e comportamentais associados ao conceito, tais como traços culturais e de personalidade que fazem a experiência ser singular a cada cliente, naquele instante de tempo, sob as condições que se apresentam em termos ambientais, tecnológicos e de relacionamento interpessoal. As experiências dos clientes configuram-se em predisposições para relacionamentos futuros com a organização e só por esta razão já merecem um tratamento diferenciado, o que é um dos propósitos na sequência desse trabalho.

Quanto a implicações para trabalhos futuros, recomenda-se que estudos empíricos sejam conduzidos em prestadores de serviços diversos para validação deste conceito proposto. Emerge a possibilidade de criar um instrumento padrão para medir a experiência de serviços que congregue as três dimensões (predisposições, interações e reações) e suas componentes.

O desafio é que o instrumento contenha itens coerentes e amparados nesta teoria, com uma métrica apropriada, para aplicá-lo indistintamente a qualquer tipo de serviço, e testar empiricamente esta abordagem. Como não é possível medir diretamente a experiência de serviços por conta de sua subjetividade, será necessário criar atributos para expressar as dimensões e suas componentes cuja relação com o que se quer medir tenha respaldo na teoria (PASQUALI, 2003).

\section{Referências}

ALLRED, C. R.; MONEY, R. B. The service hand-off: effects of multivendor service performance on customer satisfaction an experimental study. Journal of Services Marketing, v. 24, n. 2-3, p. 184-195, 2010.

ALVAREZ, D. et al. Produtividade em serviço: comunicação e competência agregando valor e fidelização no ramo hoteleiro e alimentício. Produto \& Produção, v. 9 , n. 2, 2008.
ANDERSEN, B. Business process improvement toolbox. ASQ Press, 2007.

BARON, S.; HARRIS, K. Toward an understanding of consumer perspectives on experiences. Journal of Services Marketing, v. 24, n. 7, p. 518-531, 2010.

BASSANI, D. T. et al. Qualidade nos serviços: atuação do intra-empreendedor na gestão do conhecimento. Estudo de caso em escritório de patentes. Produto \& Produção, v. 8, n. 3, 2008.

BATESON, J. E. G.; HOFFMAN, K. D. Marketing de serviços. Porto Alegre: Bookman, 2001.

BEIMBORN, D.; JOACHIM, N. The joint impact of service-oriented architectures and business process management on business process quality: an empirical evaluation and comparison. Information Systems and E-Business Management, v. 9, n. 3, p. 333-362, Sept. 2011 .

BETTENCOURT, L. A.; GWINNER, K. Customization of the service experience: The role of the frontline employee. International Journal of Service Industry Management. v. 7, p. 3-4. 1996.

BITITCI, U. S. et al. Managerial processes: business process that sustain performance. International Journal of Operations \& Production Management, v. 31, n. 7-8, p. 851-887, 2011.

BITNER, M. J.; BOOMS, B. H.; TETREAULT, M. $\mathrm{S}$. The service encounter: diagnosing favorable and unfavorable incidents. The Journal of Marketing, p. 71-84, 1990.

BITNER, M. J. et al. Customer contributions and roles in service delivery. International Journal of Service Industry Management. v. 8, p. 193-194. 1997.

BOSHOFF, C.; LEONG, J. Empowerment, attribution and apologising as dimensions of service recovery - An experimental study. International Journal of Service Industry Management. v. 9, p. 24. 1998.

BOWDLE, B. F.; GENTNER, D. The career of metaphor. Psychological review, v. 112, n. 1, p. 193, 2005.

BRIGGS, S.; SUTHERLAND, J.; DRUMMOND, S. Are hotels serving quality? An exploratory study of service quality in the Scottish hotel sector. Tourism Management, v. 28, n. 4, p. 1006-1019, 2007.

BRUNNER-SPERDIN, A.; PETERS, M. What influences guests' emotions? The case of high-quality hotels. 
International Journal of Tourism Research, v. 11, n. 2, p. 171-183, 2009.

BRUNNER-SPERDIN, A.; PETERS, M.; STROBL, A. It is all about the emotional state: Managing tourists' experiences. International Journal of Hospitality Management, v. 31, n. 1, p. 23-30, 2012.

BRYSON, J. R.; TAYLOR, M. Competitiveness by design and inimitability through service: understanding the dynamics of firm-based competition in the West Midlands jewellery and lock industries. Service Industries Journal, v. 30, n. 4, p. 583-596, 2010.

CALDAS, M. P. Paradigmas em estudos organizacionais: uma introdução à série. Revista de administração de empresas, v. 45, n. 1, p. 53-57, 2005.

CAMPENHOUDT, L.; QUIVY, R. Manual de investigação em ciências sociais. Lisboa: Gradiva, 2003.

CHANG, T. Y.; HORNG, S. C. Conceptualizing and measuring experience quality: the customer's perspective. Service Industries Journal, v. 30, n. 14, p. 2401-2419, 2010.

CHASE, R. B.; APTE, U. M. A history of research in service operations: What's the big idea? Journal of Operations Management, v. 25, n. 2, p. 375-386, 2007.

CHEN, C. F.; CHEN, F. S. Experience quality, perceived value, satisfaction and behavioral intentions for heritage tourists. Tourism Management, v. 31, n. 1, p. 29-35, Feb. 2010.

CRONIN J.R.; J. J. TAYLOR, S. A. Measuring service quality: a reexamination and extension. The Journal of Marketing, p. 55-68, 1992.

DE ROJAS, C.; CAMARERO, C. Visitors' experience, mood and satisfaction in a heritage context: Evidence from an interpretation center. Tourism Management, v. 29, n. 3, p. 525-537, Jun. 2008.

EDVARDSSON, B.; ENQUIST, B.; JOHNSTON, R. Cocreating customer value through hyperreality in the prepurchase service experience. Journal of Service Research. v. 8, p. 149-161, 2005.

FINSTERWALDER, J.; TUZOVIC, S. Quality in group service encounters A theoretical exploration of the concept of a simultaneous multi-customer co-creation process. Managing Service Quality, v. 20, n. 2, p. 109$122,2010$.

FITZSIMMONS, J. A.; FITZSIMMONS, M. J. Administração de serviços: operações, estratégias e tecnologia da informação. Porto Alegre: Bookman, 2005.

FRANCO, M. L. P. B. Análise do conteúdo. Líber Livro, 2007.

FROEHLE, C. A.; ROTH, A. V. New measurement scales for evaluating perceptions of the technologymediated customer service experience. Journal of Operations Management, v. 22, n. 1, p. 1-21, Feb. 2004.

GONÇALVES, J. E. L. As empresas são grandes coleções de processos. Revista de administração de empresas, v. 40, n. 1, p. 6-9, 2000.

GONZÁLEZ, M. E. A.; COMESAÑA, L. R.; BREA, J. A. F. Assessing tourist behavioral intentions through perceived service quality and customer satisfaction. Journal of Business Research, v. 60, n. 2, p. 153-160, 2007.

GOPALAN, R.; NARAYAN, B. Improving customer experience in tourism: A framework for stakeholder collaboration. Socio-Economic Planning Sciences, v. 44, n. 2, p. 100-112, 2010.

GROSS, M. A.; PULLMAN, M. Playing Their Roles: Experiential Design Concepts Applied in Complex Services. Journal of Management Inquiry, v. 21, n. 1, p. 43-59, Jan. 2012.

GROVE, S. J.; FISK, R. P. The impact of other customers on service experiences: A critical incident examination of "getting along". Journal of Retailing, v. 73, n. 1, p. 63-85, Spr 1997.

HE, Y.; CHEN, Q. M.; ALDEN, D. L. Consumption in the public eye: The influence of social presence on service experience. Journal of Business Research, v. 65, n. 3, p. 302-310, Mar 2012.

HEINEKE, J.; DAVIS, M. M. The emergence of service operations management as an academic discipline. Journal of Operations Management, v. 25, n. 2, p. 364-374, 2007.

HELKKULA, A. Characterising the concept of service experience. Journal of Service Management, v. 22, n. 3, p. 367-389, 2011.

HÖGSTRÖM, C.; ROSNER, M.; GUSTAFSSON, A. How to create attractive and unique customer experiences: An application of Kano's theory of attractive quality to recreational tourism. Marketing Intelligence and Planning, v. 28, n. 4, p. 385-402, 2010. 
HUANG, Y. L. et al. Impression of Liusanjie: Effect of mood on experience and satisfaction. International Journal of Tourism Research, v. 14, n. 1, p. 91-102, Jan-Feb 2012.

HUI, M. K.; BATESON, J. E. G. Perceived Control and the Effects of Crowding and Consumer Choice on the Service Experience. Journal of Consumer Research, v. 18, n. 2, p. 174-184, Sept. 1991.

HUTCHINSON, J.; LAI, F.; WANG, Y. Understanding the relationships of quality, value, equity, satisfaction, and behavioral intentions among golf travelers. Tourism Management, v. 30, n. 2, p. 298-308, 2009.

JOHNSON, M. D.; OLSEN, L. L.; ANDREASSEN, T. W. Joy and disappointment in the hotel experience: managing relationship segments. Managing Service Quality, v. 19, n. 1, p. 4-30, 2009.

JOHNSTON, R. The Zone Of Tolerance - Exploring The Relationship Between Service Transactions And Satisfaction With The Overall Service. International Journal of Service Industry Management, v. 6, n. 2, p. 46-47, 1995.

JOHNSTON, R.; KONG, X. Y. The customer experience: a road-map for improvement. Managing Service Quality, v. 21, n. 1, p. 5-24, 2011.

KIM, S. et al. Development and testing of the Consumer Experience Index (CEI). Managing Service Quality, v. 21, n. 2, p. 112-132, 2011.

KIM, J. et al. Antecedents of application service continuance: a synthesis of satisfaction and trust. Expert Systems with Applications, v. 38, n. 8, p. 9530-9542, Aug 2011.

KINGMAN-BRUNDAGE, J.; GEORGE, W. R.; BOWEN, D. E. Service Logic - Achieving Service System Integration. International Journal of Service Industry Management, v. 6, n. 4, p. 20-21, 1995.

KUMAR, V. et al. Alternative perspectives on service quality and customer satisfaction: the role of BPM. International Journal of Service Industry Management, v. 19, n. 2, p. 176-187, 2008.

KWORTNIK, R. J.; THOMPSON, G. M. Unifying Service Marketing and Operations With Service Experience Management. Journal of Service Research, v. 11, n. 4, p. 389-406, May 2009.

LADHARI, R. Service quality, emotional satisfaction, and behavioural intentions A study in the hotel industry. Managing Service Quality, v. 19, n. 3, p. 308-331, 2009.
LEVY, Y.; ELLIS, T. J. A systems approach to conduct an effective literature review in support of information systems research. Informing Science International Journal of an Emerging Transdiscipline, v. 9, p. 181212, 2006.

LOVELOCK, C. GUMMESSON, E. Whither services marketing? Journal of Service Research, v. 7, n. 1, p. 20-41, 2004.

MIKULIĆ, J.; PREBEŽAC, D. Evaluating hotel animation programs at Mediterranean sun-and-sea resorts: An impact-asymmetry analysis. Tourism Management, v. 32, n. 3, p. 688-696, Jun 2011.

MORGAN, G.; BERGAMINI, C. W.; CODA, R. Imagens da organização. Atlas São Paulo, 1996.

MARTIN-RUIZ, D.; CASTELLANOS-VERDUGO, M.; OVIEDO-GARCIA, M. D. A visitors' evaluation index for a visit to an archaeological site. Tourism Management, v. 31, n. 5, p. 590-596, Oct 2010.

NEAL, J. D.; GURSOY, D. A Multifaceted Analysis of Tourism Satisfaction. Journal of Travel Research, v. 47, n. 1, p. 53-62, Aug 2008.

PARASURAMAN, A.; ZEITHAML, V. A.; BERRY, L. L. A conceptual model of service quality and its implications for future research. The Journal of Marketing, p. 41-50, 1985.

SERVQUAL: A multiple-item scale for measuring consumer perceptions of service quality. Journal of Retailing, 1988.

PASQUALI, L. Psicometria: Teoria dos Testes na Psicologia e na Educação. São Paulo: Vozes, 2003.

PATRICIO, L.; FISK, R. P.; CUNHA, J. F. E. Designing multi-interface service experiences - The Service Experience Blueprint. Journal of Service Research, v. 10, n. 4, p. 318-334, May 2008.

PINE II, B. J.; GILMORE, J. H. Welcome to the experience economy. Harvard Business Review, v. 76, p. 97-106, 2002.

RYU, K.; HAN, H.; KIM, T. H. The relationships among overall quick-casual restaurant image, perceived value, customer satisfaction, and behavioral intentions. International Journal of Hospitality Management, v. 27, n. 3, p. 459-469, 2008.

SLACK, N. et al. Administração da produção. São Paulo: Atlas, 2006. 
SMART, P. A. MADDERN, H. MAULL, R. S. Understanding Business Process Management: Implications for Theory and Practice. British Journal of Management, v. 20, n. 4, p. 491-507, Dec. 2009.

SVARI, S. et al. A SOS construct of negative emotions in customers' service experience (CSE) and service recovery by firms (SRF). Journal of Services Marketing, v. 25 , n. $4-5$, p. $323-335,2011$.

TENG, C. C. Commercial hospitality in restaurants and tourist accommodation: Perspectives from international consumer experience in Scotland. International Journal of Hospitality Management, v. 30, n. 4, p. 866-874, Dec. 2011.

TORRES JÚNIOR, N.; MIYAKE, D. I. A participação do cliente em processos de serviço e as implicações dos possíveis papéis do cliente na criação de valor. Produto \& Produção, v. 12, n. 1, 2011.

TOSUN, C. et al. Tourist shopping experiences and satisfaction. International Journal of Tourism Research, v. 9, n. 2, p. 87-102, 2007.

VARGO, S. L.; LUSCH, R. F. Evolving to a new dominant logic for marketing. Journal of Marketing, p. 1-17, 2004.

VOORHEES, C. M. et al. It Depends Moderating the Relationships Among Perceived Waiting Time, Anger, and Regret. Journal of Service Research, v. 12, n. 2, p. 138-155, Nov 2009.

WALSHAM, G. Doing interpretive research. European Journal of Information Systems, v. 15, n. 3, p. 320-330, 2006.

WINSTED, K. F. The service experience in two cultures: A behavioral perspective. Journal of Retailing, v. 73, n. 3, p. 337-360, Fal 1997.

YEUNG, S.; LEUNG, C. Perception and attitude of Hong Kong hotel guest-contact employees towards tourists from Mainland China. International Journal of Tourism Research, v. 9, n. 6, p. 395-407, 2007.

YI, Y. GONG, T. An integrated model of customer social exchange relationship: the moderating role of customer experience. Service Industries Journal, v. 29, n. 11, p. 1513-1528, 2009.

ZEHRER, A. Service experience and service design: concepts and application in tourism SMEs. Managing Service Quality, v. 19, n. 3, p. 332-349, 2009.
Recebido em 10/04/2012. Aceito em 18/07/2012. 